\title{
A model for the shear mechanism in the enstatite-akimotoite phase transition
}

\author{
Naotaka TOMIOKA \\ Department of Earth and Planetary Sciences, Faculty of Science, Kobe University, Kobe 657-8501, Japan
}

\begin{abstract}
The shear mechanism in the clinoenstatite (space group $C 2 / c$ )-akimotoite (space group $R \overline{3}$ ) transition under high-pressure can be explained by a sweeping of partial dislocations associated with cooperative cation displacements without any long-range atomic diffusion. The shortest translation vector, [001] on the (100) plane of clinoenstatite, dissociates into a $1 / 3[001]+1 / 6[011]+1 / 3[001]+1 / 6[0 \overline{1} 1]$ vector, and the first two partial dislocations bring about a hexagonal close packed oxygen sublattice in the akimotoite structure. This shear mechanism is considered to be a favorable mechanism under high differential stress, or at high-pressures and relatively low-temperatures, where any overpressure from the hypothetical equilibrium clinoenstatite-akimotoite phase boundary would be high, but atomic diffusion would be kinetically hindered. Therefore, this mechanism would occur in subducting cold slabs in the Earth's deep mantle and in shocked meteorites.
\end{abstract}

Keywords: Akimotoite, Clinoenstatite, High-pressure transition, Shear mechanism, Dislocation

\section{INTRODUCTION}

The mechanisms of the phase transitions of silicate minerals under high-pressure play a significant role in the rheological properties and transformation kinetics in the Earth's mantle. To understand the dynamics of the Earth's interior, many researchers have studied the mechanism of the transformation of $(\mathrm{Mg}, \mathrm{Fe})_{2} \mathrm{SiO}_{4}$ olivine, which is the most abundant mineral in the upper mantle, to wadsleyite (a modified spinel structure) or to ringwoodite (a spinel structure) (e.g., Boland and Liu, 1983; Guyot et al., 1991; Fujino and Irifune, 1992; Wu et al., 1993; Brearley and Rubie, 1994; Dupas-Bruzek et al., 1998; Kerschhofer et al., 1996, 1998).

The most common mechanism in polymorphic transitions of minerals is heterogeneous nucleation and interface-controlled growth. In this mechanism, the product phase generally shows an incoherence with the reactant phase, and the shape of the resulting product phase tends to be granular. On the other hand, Poirier (1981) first proposed a shear-promoted mechanism for the olivine to ringwoodite transition. This is characterized by a diffusionless process and produces a coherent lamellar intergrowth of the resulting high-pressure phases in the host mineral. Subsequently, Boland and Liu (1983) experimentally demonstrated the occurrence of this mechanism in

doi:10.2465/jmps.060227

N. Tomioka, nao@kobe-u.ac.jp Corresponding author
$\mathrm{Mg}_{2} \mathrm{SiO}_{4}$ using transmission electron microscopy to study a recovered sample that was laser heated in a diamond anvil cell.

Pyroxene is the second most common constituent mineral of the upper mantle. The major pyroxene component, $(\mathrm{Mg}, \mathrm{Fe}) \mathrm{SiO}_{3}$, gradually changes into the garnet component $\left(\mathrm{Mg}_{3} \mathrm{Al}_{2} \mathrm{Si}_{3} \mathrm{O}_{12}\right)$ forming a majorite solid solution with increasing pressure in the mantle transition zone (Irifune and Ringwood, 1987). However, in subducted cold slabs, atomic diffusion should be more sluggish than that in the surrounding mantle. Therefore, this solution process is likely to be kinetically hindered, and low-Ca pyroxene could possibly directly transform into akimotoite $\left(\mathrm{MgSiO}_{3}\right.$-ilmenite $)$ at pressures above $20 \mathrm{GPa}$ (Hogrefe et al., 1994). In nature, akimotoite with an $(\mathrm{Mg}, \mathrm{Fe}) \mathrm{SiO}_{3}$ composition has been found in a shocked chondritic meteorite (Tomioka and Fujino, 1997, 1999). This sowed intergrowths in the host $(\mathrm{Mg}, \mathrm{Fe}) \mathrm{SiO}_{3}$ clinopyroxene, and these polymorphs exhibited a topotaxial relationship similar to that due to the shear mechanism.

So far, only one experimental study on the mechanism of the pyroxene-ilmenite transition has been reported. Hattori et al. (2001) demonstrated that $\mathrm{FeGeO}_{3}$ clinopyroxene (space group $\mathrm{C} 2 / \mathrm{c}$ ) transforms into a pseudo-ilmenite structure, $\mathrm{FeGeO}_{3}$ (II), which has been shown to have a metastable structure with a deformed hexagonal close packing of the oxygen ions at a pressure of $20 \mathrm{GPa}$ in a diamond anvil cell. On subsequent heating 
Clinoenstatite (100)
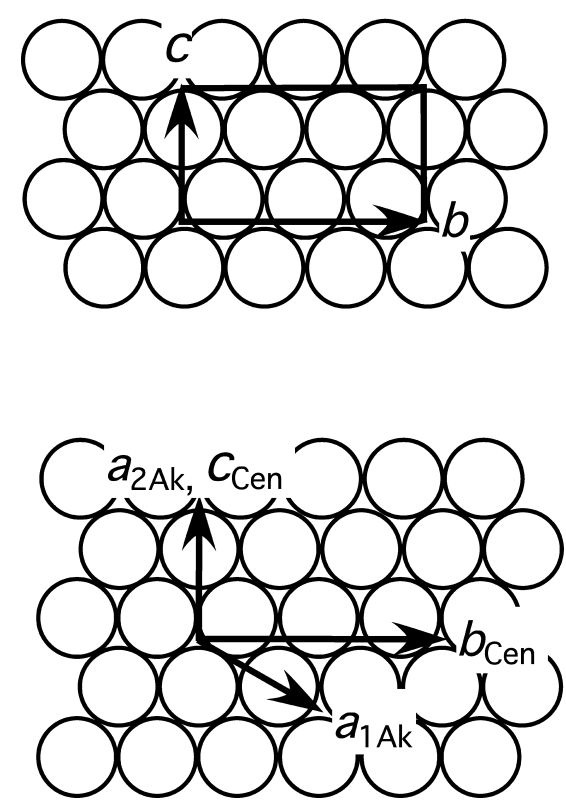

Akimotoite (0001)

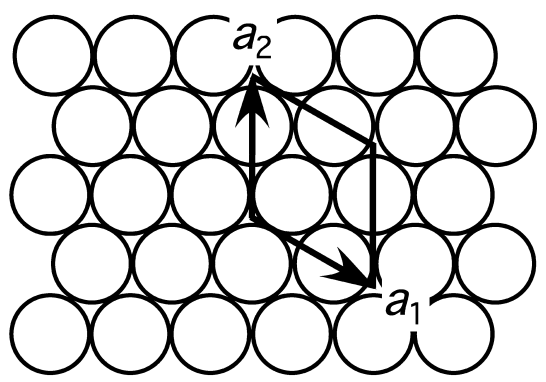

(100)Cen // (0001)Ak (010)Cen // (10̄̄0)Ak
Figure 1. The relationship between the crystallographic orientations of $C 2 / c$ clinoenstatite and akimotoite $\left(\mathrm{MgSiO}_{3}\right.$-ilmenite). The schematic drawings show the close-packed layers of oxygen ions in clinoenstatite (upper left), akimotoite (upper right), and their superimposition (bottom left). at $365{ }^{\circ} \mathrm{C}$ at a pressure of $25 \mathrm{GPa}, \mathrm{FeGeO}_{3}$ (II) inverts to form the normal ilmenite structure, and $\mathrm{FeGeO}_{3}$ (II) was not recovered at atmospheric pressure. Therefore, the crystal structure of $\mathrm{FeGeO}_{3}$ (II) was refined using the Rietveld method based on its power diffraction pattern taken at high pressure using synchrotron $\mathrm{X}^{\text {-ray radiation. }}$

Based on a comparison of the crystal structures of these $\mathrm{FeGeO}_{3}$ polymorphs, Hattori et al. (2001) suggested that the clinopyroxene to $\mathrm{FeGeO}_{3}$ (II) and $\mathrm{FeGeO}_{3}$ (II) to ilmenite transitions are promoted by a slight deformation of the oxygen layers and by a short-range movement of the cations, respectively. However, the details of the shear processes of the oxygen layers within a clinopyroxene crystal are not fully understood. When partial dislocations are introduced to this shear mechanism, a sliding of the oxygen layers in the reactant crystal occurs locally in the early stages of the transition, and this sliding gradually expands through stacking faults to encompass the entire region by a sweeping motion of the partial dislocations. This mechanism would be energetically favorable, as proposed for the olivine-ringwoodite transition in $\mathrm{Mg}_{2} \mathrm{SiO}_{4}$ (Poirier, 1981). In this work, we intended to develop an alternative model for the shear mechanism promoted by a sweeping of the partial dislocations in the clinoenstatite to akimotoite phase transition based on a topological study carried out on both structures.

\section{CLINOENSTATITE-AKIMOTOITE INTER- GROWTH IN A SHOCKED METEORITE}

The lamellar intergrowth of $(\mathrm{Mg}, \mathrm{Fe}) \mathrm{SiO}_{3}$ akimotoite with clinoenstatite was identified in the heavily shocked Tenham meteorite. $(\mathrm{Mg}, \mathrm{Fe}) \mathrm{SiO}_{3}$ perovskite, which is one of the high-pressure polymorphs of enstatite, was also discovered in this meteorite. The chemical composition of the perovskite-phase and the akimotoite were identical to that of the host clinoenstatite. These ultrahigh-pressure phases suggest that the peak pressure of the shock event had been above $22 \mathrm{GPa}$ (Tomioka and Fujino, 1997). Akimotoite has a topotaxial relationship with adjacent

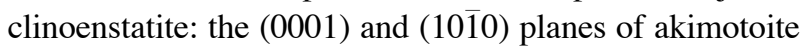
are nearly parallel to the (100) and (010) planes of clinoenstatite, respectively (see Fig. 3 in Tomioka and Fujino, 1999). These planes correspond to the layers of the nearly close-packed oxygen in both structures (Fig. 1).

These observations suggest that the akimotoite was produced in a solid-state reaction from enstatite in the host rock by the shock event. The original $\mathrm{MgSiO}_{3}-$ rich pyroxene in the host rock was orthoenstatite (space group $\mathrm{Pbca}$ ). Actually, the enstatite associated with the akimotoite is low-temperature clinoenstatite (space group $P 2_{1} / c$ ). According to high-pressure experiments on $\mathrm{MgSiO}_{3}$, the monoclinic $C 2 / c$ phase is stable at pressures above 7 GPa (Angel et al., 1992; Shinmei et al., 1999). However, this phase cannot be recovered at ambient condition, as it inverts into the $P 2_{1} / c$ phase during decompression, even at room temperature. Hence, the $P 2_{1} / c$ clinoenstatite in the Tenham meteorite is an inversion product of high-pressure $C 2 / c$ clinoenstatite formed during the post-shock pressure release. The original $\mathrm{Pbca}$ enstatite in the host rock could have initially transformed into the $C 2 / c$ phase quickly due to a displacive transition, 


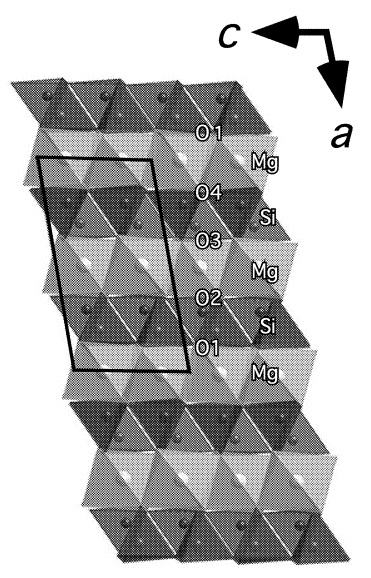

Clinoenstatite (C2/c)

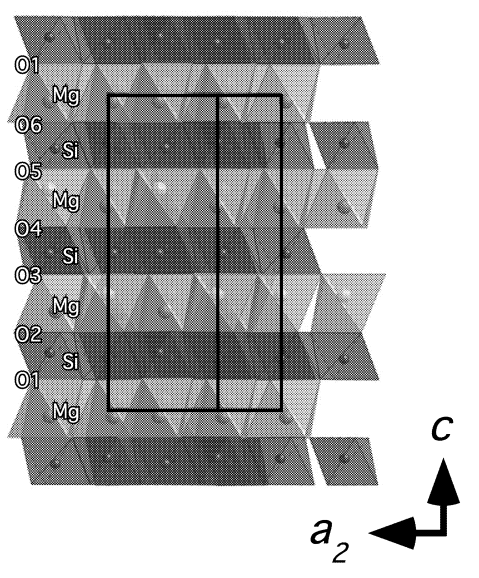

Akimotoite $(\mathrm{R} \overline{3})$
Figure 2. The crystal structures of clinoenstatite $(C 2 / c)$ and akimotoite. Both structures are composed of close packed oxygen ions, clinoenstatite (ccp), and akimotoite (hcp). The oxygen layers are denoted by O1-O6.

\section{Clinoenstatite}

$01,3,5$
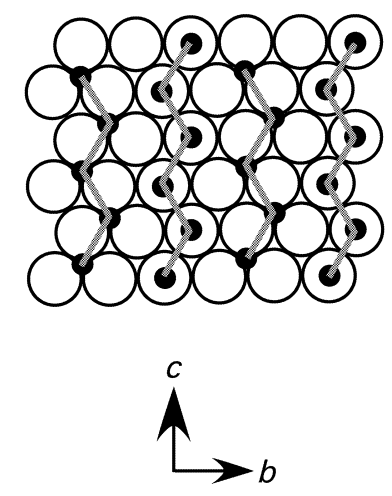

O2, 4, 6

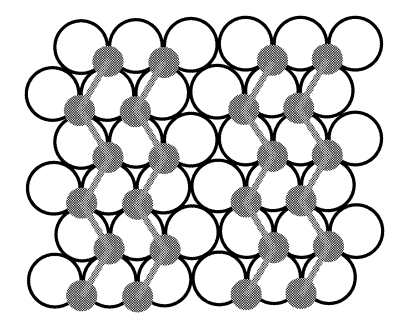

Akimotoite
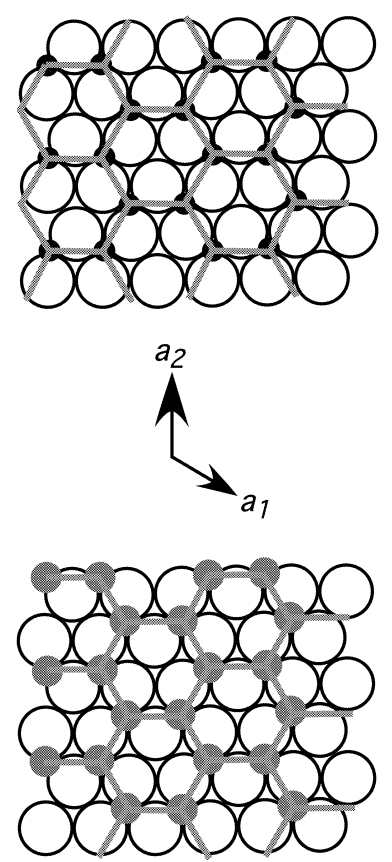

Figure 3. The simplified packing modes of clinoenstatite $(C 2 / c)$ and akimotoite. The oxygen layers are denoted by $\mathrm{O} 1-\mathrm{O} 6$. Open circles, oxygen; filled black circles, silicon; filled gray circles, magnesium. and subsequently, the $C 2 / c$ phase would then transform into akimotoite.

Hugh-Jones et al. (1996) reported frequent stacking disorders in the (100) plane of $\mathrm{FeSiO}_{3}$ clinoferrosilite produced by an orthorhombic (Pbca) to monoclinic ( $\mathrm{C} 2 / \mathrm{c})$ phase transition at a pressure of $\sim 4.2 \mathrm{GPa}$. The clinoenstatite $\left(P 2_{1} / c\right)$ in the Tenham meteorite also shows a streaking of the diffraction spots in the $a^{*}$ direction caused by stacking disorders in the (100) plane (see Fig. 3b in Tomioka and Fujino, 1999). This stacking disorder was ascribed to the $P b c a$ to $C 2 / c$ transition occurring during shock compression.

\section{A POSSIBLE MODEL FOR A SHEAR MECHA- NISM IN THE ENSTATITE-AKIMOTOITE TRANSITION}

The textural and crystallographic features of the coherent intergrowth of clinoenstatite and akimotoite found in the shocked meteorite indicate that the transition from clinoenstatite into akimotoite was caused by a shear mechanism. This transition is a diffusionless mechanism promoted by the shear of the lattices of the reactant phases. With regards to pressure-induced phase transitions in silicates, a shear mechanism was first proposed for the oliv- 

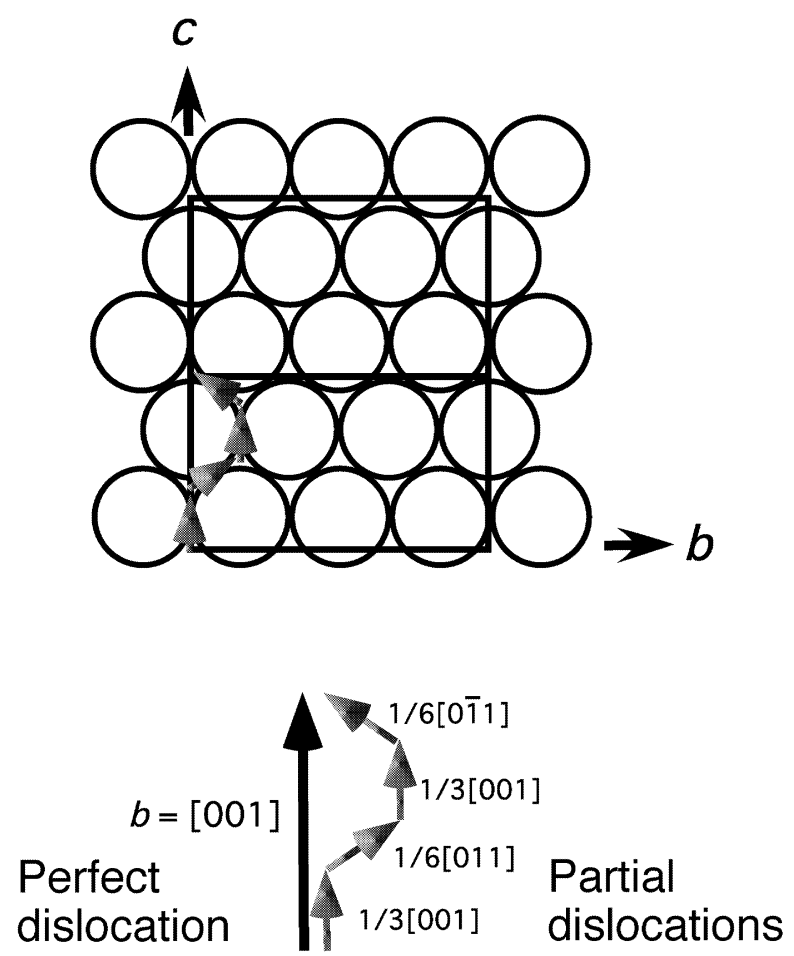

Figure 4. Dissociation of the dislocation in clinoenstatite. The open circles denote the oxygen ions on the (100) plane. The perfect dislocation, which has the smallest translational vector along the [001] direction, can be dissociated into four partial dislocations. ine-ringwoodite transition in $\mathrm{Mg}_{2} \mathrm{SiO}_{4}$. Based on a structural analysis, and using analogy to similar transitions (i.e., martensitic transformations in metals and alloys), Poirier (1981) proposed that the olivine-ringwoodite transition could be caused by a sweeping of the partial dislocations and concomitant shuffling of cations. In this paper, a possible diffusionless mechanism of the clinoenstatite $(C 2 / c)^{-}$ akimotoite transition is discussed from a topological perspective.

The crystal structures of $\mathrm{MgSiO}_{3}$ clinoenstatite $(C 2 / c)$ and akimotoite $(R \overline{3})$ are composed of cubic close packed and hexagonal close packed oxygen ions, respectively. The interstices of the close packed oxygen layers on $(100)_{\mathrm{Cen}}$ and $(0001)_{\mathrm{Ak}}$ (where the subscripts Cen and Ak denote clinoenstatite and akimotoite, respectively) are filled with alternate $\mathrm{Mg}$ and $\mathrm{Si}$ ions (see Fig. 2). During the shock event, orthoenstatite $(\mathrm{Pbca})$ transformed into high-pressure clinoenstatite $(C 2 / c)$, because this transition is rapid enough due to a displacive transition (Angel et al., 1992). Figure 3 shows the simplified cationic arrangements of clinoenstatite $(C 2 / c)$ and akimotoite. A structural change from clinoenstatite to akimotoite without any long-range atomic diffusion can be obtained by sliding the oxygen layers along with a cooperative displacement of the interstitial cations. This shear is possible by a sweeping of partial dislocations on the close packed $(100)_{\text {Cen }}$ plane.

Partial dislocations are formed by the splitting of
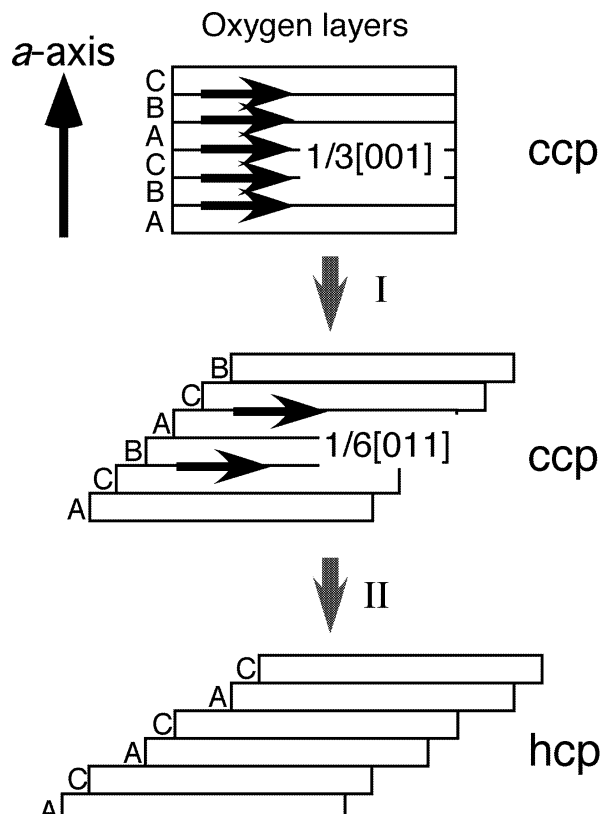

Oxygen layers

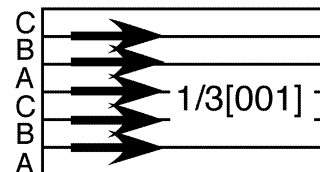

$\operatorname{ccp}$
$[001] \rightarrow \underset{\text { I }}{1 / 3[001]}+\underset{\text { II }}{1 / 6[011]}+\underset{\text { III }}{1 / 3[001]}+\underset{\text { IV }}{1 / 6[0 \overline{1} 1]}$

IAIBICIAIBIC

I

IA CIB AIC BI

II

IA CIA ClA Cl

III

$\mid \mathrm{AICIB|A|C|B|}$

IV

A B C A B C Clinoenstatite

Figure 5. The change in stacking sequence of the oxygen layers in the clinoenstatite-akimotoite transition. The cubic close packed stacking (clinoenstatite) can be converted into hexagonal close packed stacking (akimotoite) via two partial dislocations. The first shear (I) is promoted by a 1/3[001] partial dislocation between each oxygen layer. This shear maintains the cubic close packing arrangement of the oxygen atoms. The second shear (II) is promoted by a partial 1/6[011] dislocation occurring only between the oxygen layers and the Mg interstitial layers. This converts cubic close packed stacking into hexagonal close packed stacking. 
First partial
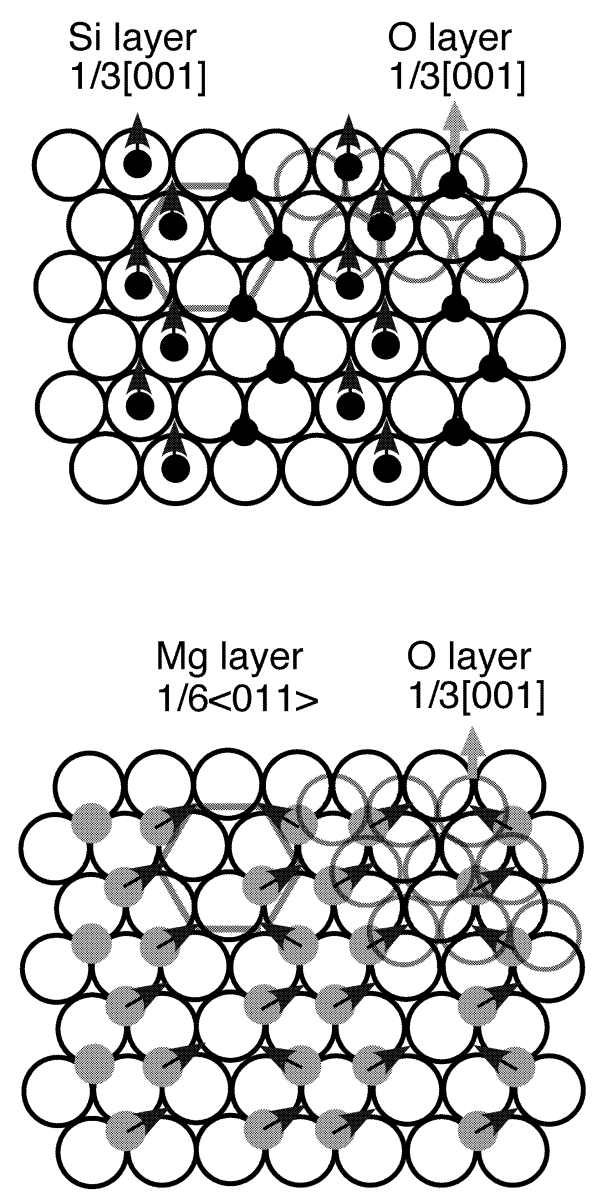

Second partial
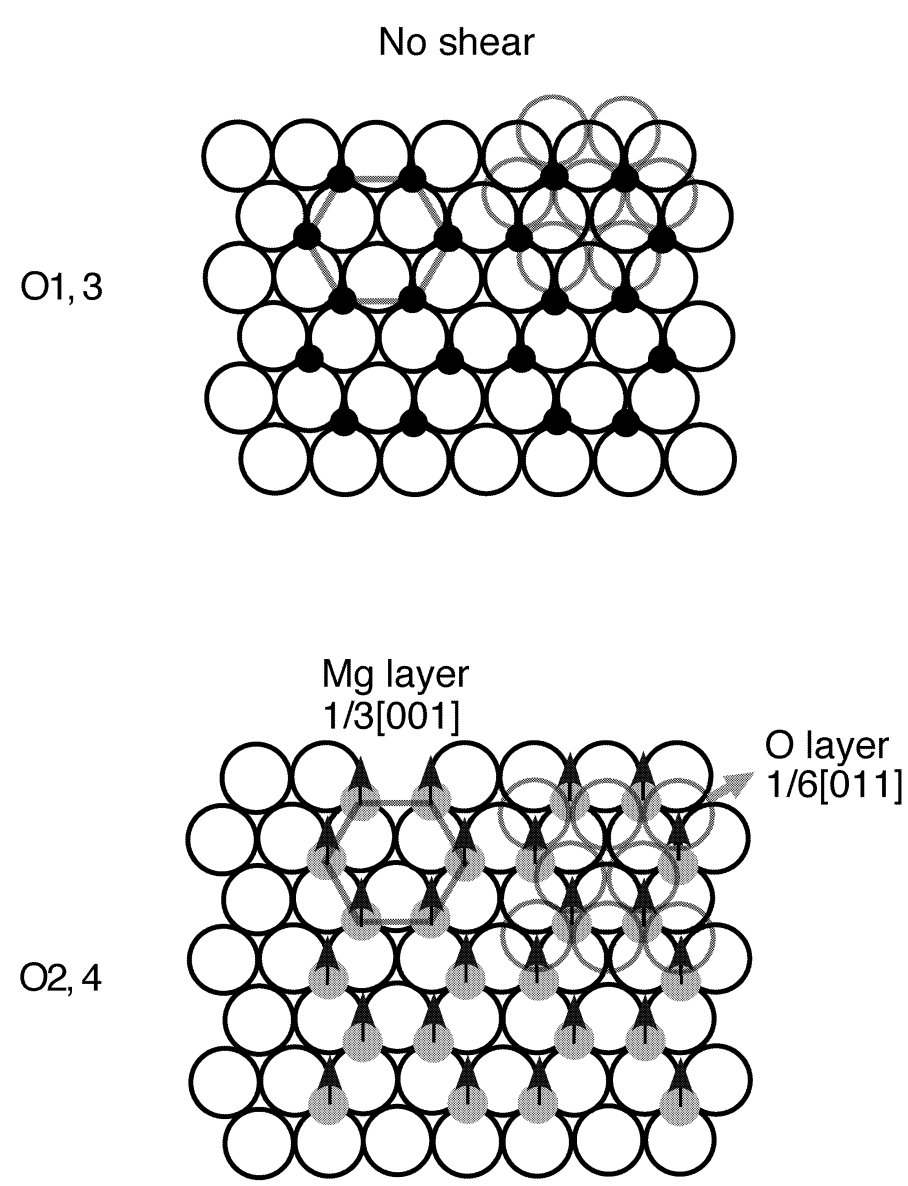

Figure 6. Synchroshear during the clinoenstatite-akimotoite transition. Open circles, oxygen ions; filled black circles, silicon ions; filled gray circles, magnesium ions; gray open circles, oxygen ions in upper layers. The arrows on the respective cations indicate the direction of movement associated with the sliding of the upper oxygen layers by the partial dislocations.

perfect dislocations, and the extended areas bound by these partial dislocations correspond to stacking faults (Putnis, 1992). Thus, the formation of partial dislocations introduces stacking faults aligned parallel to $(100)_{\mathrm{Cen}}$ during the early stage of akimotoite formation. However, it is very difficult to recognize stacking faults from the clinoenstatite-akimotoite transition, because clinoenstatite in the Tenham meteorite has frequent stacking faults on the (100) plane formed by the orthorhombic to monoclinic phase transition of enstatite, as discussed above. It is necessary that any partial dislocations are produced by the splitting of perfect dislocations for the (100)[001] slip system to shear the oxygen sublattices (Fig. 4). The [001] vector is the smallest translational vector for clinoenstatite, and it can be dissociated into four partial dislocations according to the following:

$$
[001] \rightarrow 1 / 3[001]+1 / 6[011]+1 / 3[001]+1 / 6[0 \overline{1} 1] .
$$

This reaction has been proposed previously in artificially deformed orthoenstatite (Van Duysen et al., 1985). Van Duysen et al. performed a micro-hardness test on natural orthoenstatite at room temperature, and using transmission electron microscopy, they found the dissociation of the $\mathrm{c}^{-}$-dislocation on the (100) plane had split into four partial dislocations. The dissociation of a perfect dislocation on the (100)[001] slip system into four partials may be favorable in deformation at relatively low temperatures and at high strain rates.

Figure 5 shows the changes in the stacking sequence of the oxygen layers promoted by the partial dislocations. The first partial dislocation, 1/3[001], occurs for each oxygen layer, and introduces stacking faults in the oxygen sublattices of clinoenstatite. The cubic close packed $\mathrm{ABCABC}$ stacking sequence converts into the ACBACB sequence, (which is also a cubic close packed lattice). The second partial dislocation of the alternate oxygen layers converts the sequence ACBACB into an ACACAC se- 


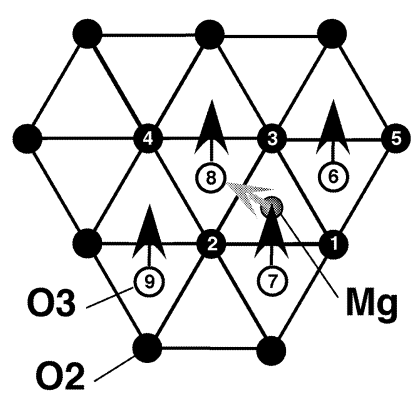

a

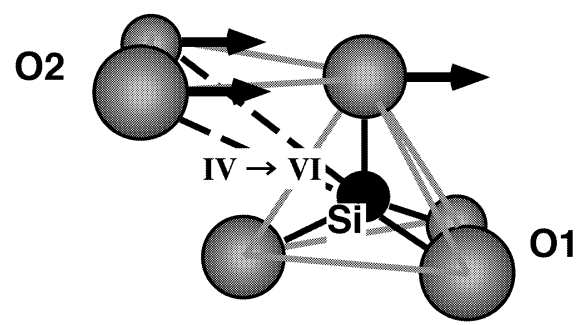

b

Figure 7. Schematic diagrams of the coordination changes of the cations with the sliding of the oxygen layers in the partial dislocations. (a) Magnesium ions (along the direction perpendicular to the $(100)_{\text {Cen }}$ direction). The solid arrows denote the displacement of the oxygen ions in the upper oxygen layer. The gray arrow indicates the relocation of the magnesium ions to the octahedral sites. The $\mathrm{Mg}-\mathrm{O}$ bonds for oxygen ions 1 and 6 are severed, and those for 4 and 9 are reconnected. (b) Silicon ions (along the direction nearly parallel to the $(100)_{\mathrm{Cen}}$ direction). The solid and broken black lines denote $\mathrm{Si}-\mathrm{O}$ bonds on the tetrahedral sites and additional $\mathrm{Si}^{-} \mathrm{O}$ bonds on the octahedral sites produced by the synchroshear, respectively.

quence, which is a hexagonal close packed lattice, corresponding to the oxygen sublattices of the akimotoite structure (Fig. 5).

Cations in the interstices of oxygen layers are supposed to move cooperatively with the shearing of the oxygen sublattices. The $\mathrm{Mg}$ and $\mathrm{Si}$ ions in the clinoenstatite structure occupy six-fold coordinated octahedral sites and four-fold coordinated tetrahedral sites, respectively (Fig. 2). In the first sweeping of the partial dislocations, if any $\mathrm{Mg}$ ions move relative to one of the oxygen layers on both sides of the $\mathrm{Mg}$ layers, then the position of the $\mathrm{Mg}$ ions change into tetrahedral sites. To avoid this coordination change, the $\mathrm{Mg}$ ions must move by jumping into neighboring octahedral sites (Fig. 6). In this case, the $\mathrm{Mg}$ ions move with displacement vectors of $1 / 6<011>$ associated with a sliding of their upper oxygen layers to form a honeycomb-like structure characteristic of akimotoite. This rearrangement process of the cations formed by jumping a single atomic distance is known as "synchroshear", and was first proposed by Kronberg (1957) for the deformation process of corundum $\left(\mathrm{Al}_{2} \mathrm{O}_{3}\right)$. On the other hand, the $\mathrm{Si}$ ions in the tetrahedral sites move along the
$1 / 3[001]$ vector relative to either oxygen layer on both sides, by an initial gliding of the partial dislocations to form a honeycomb structure consisting of six-coordinated $\mathrm{Si}$ ions. In this step, two of the six $\mathrm{Mg}-\mathrm{O}$ bonds around each individual $\mathrm{Mg}$ ion are cut, and reconnected to two other oxygen ions, and two additional $\mathrm{Si}^{-} \mathrm{O}$ bonds around individual Si ions are connected as shown in Figure 7(a, b).

In the second gliding of the partial dislocations to make a hexagonal close packed lattice, the shearing of the oxygen sublattices along the $1 / 6[011]$ direction is only produced between the oxygen layers with $\mathrm{Mg}$ interstitial layers to form the akimotoite structure. Magnesium ions in the octahedral sites forming the honeycomb structure move with a displacement vector of 1/3[001] once again to retain the six-fold coordination of the akimotoite structure (Fig. 6). After this two-step sweeping of these partial dislocations, a hexagonal close packed sublattice having cation layers composed of six-fold coordinated $\mathrm{Mg}$ and $\mathrm{Si}$ ions can be produced. This transition satisfies the crystallographic relationship where the $(0001)_{\mathrm{AK}}$ and $(10 \overline{10})_{\mathrm{AK}}$ planes are parallel to the $(100)_{\mathrm{Cen}}$ and $(010)_{\mathrm{Cen}}$ planes, respectively, as is observed between natural clinoenstatite and akimotoite in the Tenham meteorite (Fig. 1). In contrast, during the reverse transition from akimotoite to clinoenstatite, a dissociation of the perfect dislocation of the [010] plane on the (0001) slip plane occurs as follows:

$$
[010] \rightarrow 1 / 3[010]+1 / 3[110]+1 / 3[010]+1 / 3[\overline{100}] .
$$

As mentioned above, a two-step shear of the oxygen sublattices promoted by the partial dislocations in the enstatite crystal and cooperative cation displacements can cause the enstatite-akimotoite transition. This process is achieved by a minimum movement of ions and does not need any atomic diffusion beyond one unit cell dimension. This shear mechanism is considered to be a favorable mechanism under high differential stress, or under high-pressures and relatively low-temperatures, where overpressure from the hypothetical equilibrium clinoenstatite-akimotoite phase boundary is high, but atomic diffusion is kinetically hindered, as predicted in the olivineringwoodite transition (Burnley, 1995; Kerschhofer et al., 1996).

In a kinetic study on enstatite, its high-pressure transitions were observed to be much more sluggish than those of olivine (Hogrefe et al., 1994). Hogrefe et al. (1994) suggested that enstatite in cold slabs subducting into the Earth's interior would directly transform into akimotoite at pressures above $20 \mathrm{GPa}$ without breaking down to form ringwoodite + stishovite or wadsleyite + stishovite assemblages, which are stable at lower pressures than akimotoite is. A large decrease in volume $(\sim 12$ 
vol\%) in the enstatite-akimotoite transition would cause a plastic instability of the slabs. This instability would potentially result in the formation of transformational faults, and would promote a deep-focus earthquake (Hogrefe et al., 1994). If the above shear model for the enstatite-akimotoite transition is valid, then this mechanism may enhance the growth rate of akimotoite in enstatite crystals in addition to any nucleation and growth mechanisms. This enhancement may result in a rapid decrease in the volume of enstatite in a subducting slab, and cause the formation of transformational faulting in the deep mantle.

The formation mechanism of akimotoite in meteorites may depend on the temperature, pressure, and stress conditions during shock compression. The boundary conditions between the nucleation and growth and the shear mechanisms could also be a constraint on the $P-T$ history during shock metamorphism of meteorites. Therefore, further experimental studies on the enstatite-akimotoite transition are needed to understand its exact mechanism.

\section{ACKNOWLEDGMENTS}

The author acknowledges J.P. Poirier and K. Fujino for their valuable comments and K. Das for improving the manuscript. The author also wishes to thank J. Ando and T. Kubo for their constructive reviews. This work was supported by a Grant-in-Aid for Scientific Research (No. 15740316 to N.T.) and "The 21st Century COE program of Origin and Evolution of Planetary Systems" of the Ministry of Education, Culture, Sports, Science and Technology.

\section{REFERENCES}

Angel, R.J., Chopelas, A. and Ross, N.L. (1992) Stability of highdensity clinoenstatite at upper-mantle pressures. Nature, 358, 322-324.

Boland, J.N. and Liu L.G. (1983) Olivine to spinel transformation in $\mathrm{Mg}_{2} \mathrm{SiO}_{4}$ via faulted structures. Nature, 303, 233-235.

Brearley, A.J. and Rubie, D.C. (1994) Transformation mechanisms of San Carlos olivine to $(\mathrm{MgFe})_{2} \mathrm{SiO}_{4} \beta$-phase under subduction zone conditions. Physics of the Earth and Planetary Interiors, 86, 45-67.

Burnley, P.C. (1995) The fate of olivine in subducting slabs: A reconnaissance study, American Mineralogist, 80, 12931301.

Dupas-Bruzek, C., Sharp, T.G., Rubie, D.C. and Durham, W.B. (1998) Mechanisms of transformation and deformation in $\mathrm{Mg}_{1.8} \mathrm{Fe}_{0.2} \mathrm{SiO}_{4}$ olivine and wadsleyite under non-hydrostatic stress. Physics of the Earth and Planetary Interiors, 108, 3348.

Fujino, K. and Irifune, T. (1992) TEM studies on the olivine to modified spinel transformation in $\mathrm{Mg}_{2} \mathrm{SiO}_{4}$. In High-Pressure Research: Application to Earth and Planetary Sciences
(Syono, Y. and Manghnani M.H. Eds.). American Geophysical Union, Washington D.C., 237-243.

Guyot, F., Gwanmesia, G.D. and Liebermann, R.C. (1991) An olivine to beta phase transformation mechanism in $\mathrm{Mg}_{2} \mathrm{SiO}_{4}$. Geophysical Research Letters, 18, 89-92.

Hattori, T., Tsuchiya, T., Nagai, T. and Yamanaka, T. (2001) Sequential high-pressure transformations of $\mathrm{FeGeO}_{3}$ high-P clinopyroxene $(C 2 / c)$ at temperatures up to $365^{\circ} \mathrm{C}$. Physics and Chemistry of Minerals, 28, 377-387.

Hogrefe, A., Rubie, D.C., Sharp, T.G. and Seifert, F. (1994) Metastability of enstatite in deep subducting lithosphere. Nature, 372, 351-353.

Hugh-Jones, D., Sharp, T., Angel, R. and Woodland, A. (1996) The transition of orthoferrosilite to high-pressure $C 2 / c$ clinoferrosilite at ambient temperature. European Journal of Mineralogy, 8, 1337-1345.

Irifune, T. and Ringwood, A.E. (1987) Phase transformations in a harzburgite composition to $26 \mathrm{GPa}$ : implications for dynamical behaviour of the subducting slab. Earth and Planetary Science Letters, 86, 365-376.

Kerschhofer, L., Sharp, T.G. and Rubie, D.C. (1996) Intracrystalline transformation of olivine to wadsleyite and ringwoodite under subduction zone conditions. Science, 274, 7981.

Kerschhofer, L., Dupas, C., Liu, M., Sharp, T.G., Durham, W.B. and Rubie, D.C. (1998) Polymorphic transformations between olivine, wadsleyite and ringwoodite: mechanisms of intracrystalline nucleation and the role of elastic strain. Mineralogical Magazine, 62, 617-638.

Kronberg, M.L. (1957) Plastic deformation of single crystals of sapphire: Basal slip and twinning. Acta Metallurgica, 5, 507524.

Poirier, J.P. (1981) Martensitic olivine-spinel transformation and plasticity of the mantle transition zone. In Anelasticity in the Earth (Stacey, F.D., Paterson, M.S. and Nicolas, A. Eds.). American Geophysical Union, Washington D.C., 113-117.

Putnis, A. (1992) Introduction to Mineral Sciences. pp. 457, Cambridge University Press, New York.

Shinmei, T., Tomioka, N., Fujino, K., Kuroda, K. and Irifune, T. (1999) In situ X-ray diffraction study of enstatite up to 12 $\mathrm{GPa}$ and $1473 \mathrm{~K}$ and equations of state. American Mineralogist, 84, 1588-1594.

Tomioka, N. and Fujino, K. (1997) Natural (Mg,Fe)SiO ${ }_{3}$-ilmenite and -perovskite in the Tenham meteorite. Science, 277, 1084-1086.

Tomioka, N. and Fujino, K. (1999) Akimotoite, (Mg,Fe)SiO ${ }_{3}$, a new silicate mineral of the ilmenite group in the Tenham chondrite. American Mineralogist, 84, 267-271.

Van Duysen, J.C., Doukhan, N. and Doukhan, J.C. (1985) Transmission electron microscope study of dislocations in orthopyroxene $(\mathrm{Mg}, \mathrm{Fe})_{2} \mathrm{Si}_{2} \mathrm{O}_{6}$, Physics and Chemistry of Minerals, 12, 39-44.

Wu, T.-C., Bassett, W.A., Burnley, P.C. and Weathers, M.S. (1993) Shear-promoted phase transitions in $\mathrm{Fe}_{2} \mathrm{SiO}_{4}$ and $\mathrm{Mg}_{2} \mathrm{SiO}_{4}$ and the mechanism of deep earthquakes. Journal of Geophysical Research, 98, 19767-19776.

Manuscript received February 27, 2006

Manuscript accepted January 4, 2007

Published online April 3, 2007

Manuscript handled by Eiji Ohtani 